Revisão

\title{
O papel da glutamina na terapia nutricional do transplante de medula óssea
}

\author{
Silvia M. Albertini \\ Milton A. Ruiz
}

\begin{abstract}
A glutamina ( $L-G L N)$ é um aminoácido que localiza-se preferencialmente no músculo esquelético e é condicionalmente essencial nas situações onde ocorre bipercatabolismo, como no transplante de medula óssea. Nestas situações a suplementação com a L-GLN na terapia nutricional é segura e recomendada. O emprego do aminoácido com objetivo de reduzir os efeitos secundários no TMO como mucosite e manifestações digestivas parece existir. Existem dados que sugerem um efeito profilático da L-GLN em relação à doença veno-oclusiva bepática nos pacientes transplantados. O emprego do aminoácido em combinação com anti-oxidantes, o uso do mesmo via enteral e/ou parenteral, são respostas que devem ser obtidas através de estudos em grupos homogêneos e selecionados de pacientes submetidos ao transplante de medula óssea.

Rev.bras.hematol.hemoter., 2001, 23(1): 41-47
\end{abstract}

Palavras-chave: Glutamina, terapia nutricional, transplante de medula óssea

\section{Introdução}

O transplante de medula óssea (TMO) é um procedimento utilizado para o tratamento de doenças onco-hematológicas, com a finalidade de reconstituição do sistema hematopoético por meio da transferência de células pluripotentes ao paciente. O TMO requer uma ablação inicial da medula óssea do paciente através de quimioterapia citorredutora de altas doses, ou radioterapia (1). Recentemente, o procedimento tem sido aplicado também em doenças genéticas e em portadores de tumores sólidos (2). Na seqüência, dependendo da origem das células, retiradas do próprio paciente ou de um doador HLA-compatível ou não, o procedimento receberá a denominação de autogênico ou alogênico.

Classicamente para o TMO, a procedência das células é de crista ilíaca da medula óssea, obtida em ambiente cirúrgico ou então oriundas do sangue periférico e coletadas em máquinas processadoras de aférese (3). Este último procedimento é mais recente e cada vez mais freqüente, porque oferece inúmeras vantagens ao doador quando comparado à coleta tradicional de medula óssea. A obtenção das células pode ocorrer diretamente da medula óssea simplesmente, ou com a expansão e mobilização das células com fatores de crescimento hematopoéticos, associados ou não à quimioterapia (2).

Hospital de Base de S.J do Rio Preto, Serviço de Nutrição

Unidade de TMO HB-FUNFARME / CINTRANS

1 - Nutricionista da Faculdade de Medicina de São José do Rio Preto, SP

2 - Coordenador da Unidade de TMO / Hospital de Base de São José do Rio Preto, SP

Correspondência para: Milton Artur Ruiz

Faculdade de Medicina de São José do Rio Preto, SP

Hospital de Base de São José de Rio Preto. Av Brigadeiro Faria Lima, 5416. CEP: 15090-O00. São José do Rio Preto, SP 
A partir do momento em que as células estão disponíveis para infusão, o paciente então é submetido a uma nova quimioterapia de altas doses (regime de condicionamento), para maximizar a destruição do tecido anormal e mielóide. A irradiação corporal total é adicionada também com freqüência nos programas de condicionamento.

Em todo o procedimento de preparação (obtenção de células e regime de condicionamento), durante a internação, os pacientes apresentam toxicidade orgânica intensa e variável, além de leucopenia e plaquetopenia extrema durante vários dias no período imediato do TMO. Este é o período de maior vulnerabilidade para os pacientes desenvolverem complicações infecciosas precoces (2). No TMO alogênico comumente ocorre uma interação imunológica entre as células do doador e tecidos do hospedeiro, podendo ocorrer a qualquer tempo após o procedimento a doença do enxerto contra o hospedeiro (DECH). O risco de toxicidade nos pacientes diminui no momento em que as células infundidas estão implantadas.

As complicações precoces e relacionadas à toxicidade da quimio/radioterapia incluem náusea, vômito, diarréia, mucosite, anorexia e fraqueza muscular que afetam o estado nutricional do paciente e interferem na ingestão oral de alimentos (4). Com o objetivo de minimizar as complicações, reduzir o tempo de hospitalização, normalizar a resposta imunológica, incrementar a ingestão alimentar e a atividade do paciente, manter ou melhorar o estado nutricional, todos os pacientes necessitam de terapia de suporte (5).

O aminoácido glutamina (L-GLN) é conhecido por aumentar a resposta imunológica $(6,7)$, facilitar o crescimento e reparar a mucosa intestinal em seguida a radioterapia e a medicamentos que danificam a mucosa $(8,9$, 10). A L- GLN tem também a função de otimizar o balanço nitrogenado $(11,12)$ e manter a síntese de proteína muscular (13). Estes dados sugerem que a suplementação com L- GLN pode desempenhar um papel importante na terapia nutricional dos pacientes submetidos ao TMO. Em relação a esta assertiva existem dados que demonstram os efeitos benéficos da L-GLN nos pacientes $(2,14,15)$, sendo o objetivo deste texto discutir e apresentar os dados relevantes e as dúvidas existentes quanto ao emprego do aminoácido na terapia nutricional dos pacientes submetidos ao TMO.

\section{O que é a Glutamina?}

A glutamina (L-GLN) é um aminoácido neutro, glicogênico, dispensável em condições normais e sintetizado por vários tecidos orgânicos. É o aminoácido mais abundante no sangue e no pool de aminoácidos livres e intracelulares (70 - 80\%) (16). A concentração de L-GLN no músculo esquelético é 30 vezes maior que a observada no sangue, e o mesmo considerado o principal local de estoque e síntese deste aminoácido (17).

A L-GLN é uma fonte energética importante para os macrófagos, linfócitos e demais células do sistema imunológico (18). Os macrófagos e os linfócitos utilizam a L- GLN de forma semelhante à utilização da glicose. Este aminoácido estimula a proliferação de linfócitos e diferenciação das células B, produção de IL-1 e a fagocitose dos macrófagos (16).

O aumento da demanda de glutamina nos tecidos resulta na redução significativa dos seus níveis plasmáticos, mesmo ocorrendo aumento na liberação de glutamina pelos músculos esqueléticos. Por isto, a L-GLN é considerada um aminoácido condicionalmente essencial em situações de hiper-catabolismo associadas a grandes cirurgias, queimaduras extensas, sepse e inflamações, onde existe balanço nitrogenado negativo e elevação das taxas de degradação muscular (19). A L-GLN é fonte importante de energia para os enterócitos e para a integridade e função da mucosa intestinal. Deste modo, o aminoácido faz parte da terapia nutricional das patologias intestinais com o objetivo de preservar estruturalmente a parede das mucosas do aparelho digestivo (20). Ela é uma reguladora da síntese protéica e da uréia e transporta a amônia da periferia para os órgãos viscerais.

É considerada também, uma precursora da biossíntese dos ácidos nucléicos e de todas as células do organismo (21). As fontes alimentares da L-GLN disponíveis estão nas carnes, ovos, derivados do leite e da soja (16). 


\section{Estudos clínicos com a L-GLN no TMO}

O estudo clínico inicial sobre a eficácia da L-GLN foi realizado por Ziegler (22), que demonstrou a sua segurança clínica e determinou a dose-resposta das soluções de nutrição parenteral que continham o aminoácido $(22,23)$.

As indicações do uso e suplementação com a L- GLN na terapia nutricional em diversas entidades estão bem documentadas e incluem as patologias digestivas, pacientes críticos ou convalescentes de queimaduras, traumas, choque séptico e nos submetidos ao transplante de medula óssea. Na tabela 1 sumarizamos as principais indicações terapêuticas da L-GLN $(12,24)$.

A eficácia metabólica da L-GLN no transplante de medula óssea advém do estudo de Ziegler (23), no qual foram avaliados 45 pacientes, portadores de diversas hemopatias malignas. Os pacientes foram randomizados para receberem ou não a L-GLN na nutrição parenteral total (NPT). A solução utilizada no experimento continha $0,57 \mathrm{~g}$. de L-GLN/kg de peso corporal/dia.

Tabela 1. Indicações terapêuticas do uso da $L-G L N$

- Disfunções intestinais

- Síndrome do intestino curto

- Doença inflamatória intestinal

- Enterite induzida por radio/ quimioterapia

- Enterite infecciosa

- Estados de Imunodeficiência

- AIDS

- Disfunção do sistema imune associada a estados críticos ou ao transplante de medula óssea

- Situações de hipercatabolismo

- Queimaduras/trauma múltiplo/ cirurgias de grande porte

- Choque séptico

- Transplante de medula óssea

- Outros estados críticos

Fonte: Adaptado de Furst P et al ${ }^{(12)}$ e Savy $G^{(24)}$
Os resultados do balanço nitrogenado de 23 pacientes da casuística entre os dias 4 e 11 do TMO foram melhores no grupo suplementado com L-GLN, em relação aos que não a utilizaram. A freqüência de colonização microbiana foi significativamente menor nos pacientes que receberam a L-GLN, ocorrendo também um número reduzido de infecções no grupo suplementado. A permanência hospitalar dos pacientes suplementados foi reduzida (X $29 \pm 1$ dias / $36 \pm 2$ dias) em 7 dias, quando comparada ao grupo não suplementado.

Outros estudos realizados pelo mesmo autor e colaboradores, encontraram resultados também em relação ao custo hospitalar. Os custos hospitalares foram de US\$21,095 menores nos pacientes suplementados quando comparados aos custos dos pacientes com a terapia padrão (25). Os benefícios fisiológicos adicionais encontrados em outros estudos incluíram a atenuação da expansão de líquido extracelular (26), a melhora no humor (27), e aumento da recuperação dos linfócitos circulantes, particularmente os linfócitos-T (28).

Um protocolo semelhante ao de Ziegler, foi aplicado em 29 pacientes portadores de hemopatias malignas e tumores sólidos e submetidos ao transplante de medula óssea (29). Neste estudo duplo-cego, randomizado, os pacientes submetidos a TMO autólogos e alogênicos receberam nutrição parenteral total (NPT) isocalórica e isonitrogenada até o momento em que passaram a consumir por via oral $50 \%$ de suas necessidades estimadas. Observou-se em 10 pacientes que a água corporal total e a água extracelular aumentaram nos pacientes que receberam NPT padrão e diminuram significativamente nos pacientes que receberam NPT suplementada com a LGLN. A freqüência de culturas bacterianas positivas, infecções clínicas e mortalidade não diferiu significativamente entre os 2 grupos. Não houve diferenças significativas em relação ao diagnóstico.

Um outro estudo (30) foi realizado com 50 pacientes não selecionados, submetidos ao TMO, recebendo 50 gr. do dipeptídeo glicil- glutamina (38 g de L-GLN) na NPT e foi observado que o grupo suplementado apresentou redução das manifestações digestivas. 
A proporção lactulose/manitol, uma medida da permeabilidade do intestino delgado também foi superior no grupo suplementado. Observou-se também neste estudo menos dias de febre. Com estes resultados os autores concluíram pelo benefício da L-GLN no trato gastrointestinal dos pacientes transplantados.

A mucosite, ou inflamação da cavidade oral, é uma complicação dolorosa associada com a quimioterapia em alta dose e tem sido um ponto observado em grande número de estudos. Em dois destes estudos $(23,29)$, a atenuação da mucosite com o emprego da L-GLN administrada por via endovenosa não foi observada. Um outro estudo (31) com a L-GLN via oral (16 gr./dia administrada em 4 doses diárias) não observou a atenuação da mucosite induzida pela quimioterapia. Apesar destes resultados, outros estudos têm preconizado o uso mais intensivo da L-GLN oral no tratamento da mucosite durante o TMO (32). Cento e noventa e cinco pacientes foram randomizados para receberem um bochecho oral, com deglutição posterior, durante o regime de condicionamento até $28^{\circ}$ dia do transplante. A dose foi $1 \mathrm{~g} . / \mathrm{M}^{2}$ administrada 4 vezes por dia. O grupo controle recebeu glicina e o grupo experimental recebeu L-GLN. Os aminoácidos foram dissolvidos no mesmo veículo e as duas suspensões eram visualmente idênticas. No estudo observou-se a duração e quantidade de opiáceo administrado para reduzir a dor associada com a mucosite e a sensação de mucosite relatada pelo paciente.

No grupo suplementado com L- GLN houve melhora destes parâmetros.

Não houve nenhuma diferença em relação a suplementação nos pacientes que apresentaram doença do enxerto-contrahospedeiro (DECH). Entretanto, a taxa de sobrevida nos suplementados aos 28 dias do TMO era melhor no grupo suplementado, diferença esta que não se observou no $100^{\circ}$ dia do transplante.

A avaliação da L-GLN parenteral e enteral em pacientes submetidos ao TMO foi relatada por Schloerb and Skikne (33). Em um estudo randomizado com 66 pacientes $(43 \mathrm{com}$ hemopatias malignas, 23 com tumores sólidos 21 com câncer de mama) receberam L-GLN oral 35 pacientes, L -Glicina 31, doses similares nos dois grupos de 10 g. três vezes ao dia. Quando a NPT tornou-se necessária, os pacientes do grupo da L- GLN receberam adicionalmente através da NPT, L-GLN na dose de $0,57 \mathrm{gr} / \mathrm{kg} /$ dia EV. O grupo da L-Glicina recebeu a NPT padrão isocalórica e isonitrogenada. Os pacientes com hemopatias malignas receberam quimioterapia de alta dose e ou radioterapia, sendo que 18 foram submetidos ao TMO alogênico e 25 ao autólogo. Os pacientes com tumores sólidos receberam transplantes autólogos. Houve 14 óbitos nos pacientes do estudo durante o período de internação. Comparando-se os pacientes do TMO autólogo e alogênico, em relação aos grupos suplementados com L-Glicina e L- GLN, não foi observada diferença significativa em relação aos parâmetros de permanência hospitalar global, dias de NPT, tempo de recuperação dos neutrófilos, hemoculturas positivas, sepse, mucosite e de diarréia. A DECH ocorreu em 1 dos 10 pacientes hematológicos que recebiam L- GLN e em 3 dos 8 pacientes que recebiam LGlicina. Este resultado também não foi considerado estatisticamente significativo. A provável redução da NPT no grupo de L-GLN observada no estudo, sugere um aumento da sobrevida de longo prazo destes pacientes quando comparados ao do grupo da L-Glicina. Embora tenha sido sugerido que a L-GLN parenteral e oral combinada produza um benefício limitado, dado este sugerido no estudo para os pacientes com hemopatias malignas ou tumores sólidos, estudos com a L-GLN em grupos mais homogêneos de pacientes sugerem esta associação (2).

Os pacientes que recebem quimioterapia de alta dose no TMO podem desenvolver uma forma de falência hepática denominada doença veno-oclusiva hepática. A alteração é devido a edema sub-endotelial, redução do calibre das veias centrais e obstrução do fluxo hepático. A alteração pode estar relacionada à agressão de radicais livres e a deficiência de antioxidantes (2). A L-GLN tem sido considerada importante para a produção de glutationa intestinal ou hepática durante o estresse catabólico (34). A glutationa intracelular é um tripeptídeo e um dos antioxidantes mais abundante encontrado no organismo. Neste ponto queremos ressaltar 
que as soluções de NPT padrão empregada na maioria dos pacientes não contém L-GLN e isto pode causar estresse oxidativo associado a depleção de glutationa, através de mecanismos desconhecidos (35). Relatos de casos $(36,37)$ sugerem que a administração de GLN e vitamina E (um antioxidante altamente ativo localizado nas membranas celulares) pode ser útil na solução deste problema grave.

Outro estudo recente (38) relatou que a NPT enriquecida com o dipeptídeo glicil-L-GLN preservou significativamente os níveis plasmáticos de albumina e proteína $\mathrm{C}$ em pacientes submetidos ao TMO, o que sugere uma proteção da função hepática e redução do risco da doença veno-oclusiva. Em relação a este dado, outros estudos são necessários para determinar se a profilaxia com L-GLN e vitamina E, ou outras combinações de antioxidantes reduzem a freqüência desta complicação.

Recentemente foi relatada a existência de interações fármaco-cinéticas entre a L-GLN e o Metrotexate. Especula-se que a L-GLN inibe o clearance renal do metotrexate expondo o hospedeiro a doses maiores da droga (39). Em contrapartida, outros estudos demonstram que a L-GLN aumenta o nível e a concentração do metotrexate em tumores experimentais. Assim sendo, melhoraria o efeito tumoricida do antimetabólito (40).

\section{Conclusão}

A administração de GLN parenteral ou enteral, seja como aminoácido livre ou na forma de dipeptídeo parece ser segura e eficaz nos pacientes submetidos ao TMO. Embora nem todos os estudos demonstrem benefícios claros, existem dados positivos suficientes para sugerir que este nutriente deva ser considerado suporte metabólico de muitos, se não de todos, os indivíduos submetidos ao processo catabólico decorrente do TMO. Esta assertiva, como demonstrado anteriormente e nos estudos recentes com L-GLN e em outros grupos de pacientes críticos, corrobora que a suplementação da L-GLN na terapia de suporte nutricional reduz a morbidade e a mortalidade por infecção (7, 41, 42). São necessários, no entanto, novos estudos clínicos controlados com a nutrição suplementada com a L-GLN combinadas com antioxidantes, com o objetivo de se avaliar a importância do aminoácido nas complicações precoces do TMO.

\section{The role of glutamine in nutritional support of bone marrow transplantation}

Silvia M. Albertini, Milton A. Ruiz

\begin{abstract}
Glutamine is an amino acid which is usually found in the skeletal muscles and it is conditionally essential where there is byper cathabolism as in bone marrow transplantation. In these situations nutritional support therapy using L-GLN supplements is both safe and recommended. There seems to be a use for amino acid with the goal of reducing the secondary effects, such as mucositis and digestive tract manifestations, of these transplants. There are data which suggest a prophylactic effect of the LGLN in relation to hepatic veno-occlusive disease in transplant patients. The utilisation of amino acid in combination with antioxidants either by enteral or parenteral means, are questions which should be answered through further study of selected and beterogeneous groups of bone marrow transplant patients.

Rev.bras.hematol.hemoter., 2001, 23 (1): 41-47
\end{abstract}

Key words: Glutamine, nutritional therapy, bone marrow transplantation

\section{Referências bibliográficas:}

1. Duncombe A. ABC of clinical haematology: Bone marrow and stem cell transplantation. BMJ 1997; 314:1179.

2. Wilmore, DW, Schloerb PR, Ziegler TR. Glutamine in the support of patients following bone marrow transplantation. Curr Opin Clin Nutr Metab Care 1999; 2: 323-327.

3. Ruiz MA, Zola PA, Homsi CM, Borim LNB, Garcia AA, Mota RS et al. Relato de nove casos submetidos a transplante de medula óssea. Hospital de Base de São José do Rio Preto, São Paulo. NewsLab 2000; 7 (42): 100.

4. Abib SRR, Azevedo AM, Azevedo WM, et al. Conduta nutricional em receptores de 
transplante de medula óssea. Parte I: Atenção hospitalar. Bol Soc Bras Hematol Hemot 1996; 18(172): 57- 60.

5. Bergerson SL. Nutritional Support in Bone Marrow Transplant Recipients. In: On call in... Bone Marrow Transplantation. Burt RK, Deeg HS, Lothian ST, Santos GW. RG Lander Company and Chapman \& Hall, Canada; 1996. P.343-55.

6. Wilmore DW, Shabert JS. Role of glutamine in immunologic responses. Nutrition 1998; 14: 618-626.

7. Houdijk APJ, Rijnsburger ER, Jansen J, Wesdorp RIC, Weiss JK, McCamish MA, et al. Randomized trial of glutamine-enriched enteral nutrition on infections morbidity in patients with multiple trauma. Lancet 1998; 352: 772-776.

8. Van der Hulst RRWJ, Van Kreet BK, Von Meyenfecht, Brummer RJ, Arends JW, Deutz $\mathrm{NE}$, Soeters PB. Glutamine and the preservation of gut integrity. Lancet 1993; 334: 1363-1365.

9. Klimberg VS, Souba WW, Dolson DJ . Prophylactic glutamine protects the intestinal mucosa from radiation injury. Cancer 1990; 60: 62-68.

10. Bai MX, Jiang ZM, Liu YW et al. Effects of alanyl-glutamine on gut barrier function. Nutrition 1996; 12 (11/12): 793 - 796.

11. Merlien BJ, Stehle P, Waehtler P, Sledheff HP, Keller M, Kenig W et al. Totalparenteral nutrition with glutamine dipeptides after major abdominal surgery. Ann Surg 1998; 227: 302-308.

12. Furst P, Pogan K, Stehle P. Glutamine dipeptides in clinical nutrition. Nutrition 1997; 13(7/8): 731-737.

13. Hammarqvist F, Wernerman J, Ali MR, Von der Decken A, Vinners E. Addition of glutamine to total parenteral nutrition from elective abdominal surgery spares free glutamine in muscle, counteracts the fall in muscle protein synthesis, and improves nitrogen balance. Ann Surg 1989; 209: 455-461.

14. Ziegler TR, Smith RJ, Byrne TA et al. Potential role of glutamine supplementation in nutritional support. Clin Nutr 1993; 12 (suppl 1) S82-S90.

15. Griffiths RD. Glutamine: Establishing clinical indications. Curr Opin Clin Nutr Metab Care 1999; 2: 177-182.

16. Borges VC, Duarte AJS, Waitzberg DL. Imunonutrição: Uma perspectiva. Capítulo 58. In: Waitzberg DL. Nutrição Enteral e Parenteral na Prática Clínica. $2^{-}$Edição, 1995; Editora Atheneu. 642 p.

17. Souba WW, Klimberg VS, Plumley DA, et al. The role of glutamine in maintaining a bealthy gut and supporting the metabolic response to injury and infecction. J Surg Res 1990; 48: 383-391.

18. Curi R, Newsholme P, Pithon-Curi PC, et al. Metabolic fate of glutamine in lymphocytes, macrophages and neutrophils. Bras J Med Biol Res 1999; 32 (1):15-21.

19. Padovese R, Lima MR, Martins AKA et al. Glutamina: um aminoácido "condicionalmente essencial". Laes \& Haes 2000; 21 (124) 124-138.

20. Campos F G, Waitzerberg D L, Logulo $\mathrm{AF}$ et al. Importância da glutamina na prática clínica Arq. Gastroenterol. 1996; 33 (2): 86-92.

21. Ziegler TR. Glutamine supplementation in catabolic ilness. Am J Clin Nutr 1996; 64: 645-647.

22. Ziegler TR, Benfell K, Smith RJ, Young LS, Brown E, Ferrari-Baliviera et al. Safety and metabolic effects of L-glutamine administration in bumans. J Parenter Enter Nutr 1990; 14 (suppl): 1375-1465.

23. Ziegler TR, Young LS, Benfell K, Scheltinga M, Hortos K, Bye R, et al. Clinical and metabolic efficacy of glutamine-supplemented parenteral nutrition after bone marrow transplantation.

Ann Int Med 1992; 116: 821-828.

24. Savy GK. Enteral glutamine supplementation: clinical review and practical guideliness NCP 1997; 12 (6): 259 - 262.

25. MacBurney M, Young LS, Ziegler TR, Wilmore DW. A cost-evaluation of glutamine-supplemental parenteral nutrtion in adult bone marrow transplant patients. $\mathbf{J}$ Am Diet Assoc 1994; 94: 1263- 1266.

26. Scheltinga M, Young LS, Benfell K, Bye R, Ziegler TR, Santos A, et al. Glutamineenriched intravenous feedings attenuate extracellular fluid expansion after surgical stress. Ann Surg 1991; 214: 385- 393. 
27. Young LS, Bye RL, Scheltinga M, Ziegler TR, Jacobs DO, Wilmore DW. Patients receiving glutamine-supplemented intravenous feedings report na improvement in mood. J Parenter Enter Nutr 1993; 17: 422-427.

28. Ziegler TR, Bye RL, Persinger RL, Young LS, Antin JH, Wilmore DW. Effects of glutamine supplementation in circulating lymphocytes after bone marrow transplantation: a pilot study. Am J Med Sci 1998; 315: 4-10.

29. Schloerb PR, Amare M. Total parenteral nutrtion with glutamine in bone marrow transplantation and other clinical applications (a randomized double -blind study). $\mathbf{J}$ Parenter Enter Nutr 1993; 17: 407-413.

30. Poynton $\mathrm{CH}$, Maughan T, Elia M. Glycil Lglutamine reduces gut toxicity in bone marrow transplantation [abstract]. Blood 1995; 86: 586.

31. Jebb AS, Marcus R, Elia M. A pilot study of oral glutamine supplementation in patients receiving bone marrow transplantation. Clin Nutr 1995; 14: 162-165.

32. Anderson PM, Ramsay NRC, XO S, Rydholm $\mathrm{N}$, Rogosheske J, Nicklow R, et al. Use of low dose oral glutamine on painful stomatitis during bone marrow transplantation. Bone Marrow Transplant 1998; 22: 339-344.

33. Schloerb PR, Skikne BS. Oral and parenteral glutamine in bone marrow transplantation. $A$ randomized double-blind study. $\mathbf{J}$ Parenter Enter Nutr 1999; 23: 117-122.

34. Cao, Feng Z, Hoos A, Klimberg VS. Glutamine enhances gut gluthatione production. $\mathbf{J}$ Parenter Enter Nutr 1998; 22: 224- 227.

35. Jonas CR, Ziegler TR. Nutrition support and antioxidant defenses: a cause for concern?
Am J Clin Nutr 1998; 68: 765-767.

36. Nattakon TU, Charlton A, Wilmore DW. Use of vitamin E and glutamine in the successful treatment of severe veno-occlusive disease following bone marrow transplantation. Nutr Clin Pract 1995; 10: 16-18.

37. Goringe AD, Brown S, O'Callaghan VO, Rees J, Jebb S, Elia M, Poynton CH. Glutamine and vitamin $E$ in the treatment of hepatic veno-occlusive disease following high -dose chemotherapy. Bone Marrow Transplant 1998; 21: 829-832.

38. Brown AS, Goringe A, Fegan C, Davies SV, Giddings J, Whittaker JA, et al. Parenteral glutamine protects bepatic function during bone marrow transplantation. Bone Marrow Transplant 1998; 22:181-189.

39. Charlmand SL, Bartlett DL, Torosian MH. A significant methotrexate-glutamine pharmacokinetics interaction. Nutrition 1995; 11:154-158.

40. Rubio IT, Cao Y, Hutchins LF, Westbrook $\mathrm{KC}$, Klimberg VS. Effect of glutamine on methotrexate efficacy and toxicity. Ann Surg 1998; 227:772-780.

41. Neu J, Roig JC, Meetze WH, Veerman M, Carter C, Milisaps M, et al. Enteral glutamine supplementation for very low birth weight infants decreases morbidity. J Pediatr 1997; 131: 691-699.

42. Griffiths RD, Jones C, Palmer TE. Six-month outcome of critically ill patients given glutamine- supplemented parenteral nutrition. Nutrition 1997; 13: 295- 302.

Recebido: 15/01/01

Aceito: 30/03/01 